\title{
Digital radiography detector performance
}

Luís Lança and Augusto Silva

\begin{abstract}
The characterization of physical properties of digital imaging systems requires the determination and measurement of detectors' physical performance. Those measures such as modulation transfer function (MTF), noise power spectra (NPS), and detective quantum efficiency (DQE) provide objective evaluations of digital detectors' performance.

To provide an MTF, NPS, and DQE calculation from raw-data images it is necessary to implement a method that is undertaken by two major steps: (1) image acquisition and (2) quantitative measure determination method. In this chapter a comprehensive description about a method to provide the measure of performance of digital radiography detectors is provided.
\end{abstract}

Keywords: Digital radiography; Detector; Performance; Physical performance; Measurement; Modulation transfer function; Noise power spectra; Detective quantum efficiency; Objective; Image acquisition; Quantitative measure. 